\title{
Comparison of the Radio Frequency Interference (RFI) in the Region of Solar Burst Type III Data At Selected CALLISTO Network
}

\author{
Z. S. Hamidi ${ }^{1, *}$, N. N. M. Shariff ${ }^{2}$, C. Monstein ${ }^{3}$ \\ ${ }^{1}$ School of Physics and Material Sciences, Faculty of Sciences, MARA University of Technology, \\ 40450, Shah Alam, Selangor, Malaysia \\ ${ }^{2}$ Academy of Contemporary Islamic Studies (ACIS), MARA University of Technology, \\ 40450, Shah Alam, Selangor, Malaysia \\ ${ }^{3}$ Institute of Astronomy, Wolfgang-Pauli-Strasse 27, Building HIT, Floor J, \\ $\mathrm{CH}-8093$ Zurich, Switzerland \\ *E-mail address: zetysh@salam.uitm.edu.my
}

\begin{abstract}
Compact Astronomical Low-frequency, Low-cost Instrument for Spectroscopy in Transportable Observatories (CALLISTO) is a global network of spectrometer system with the purpose to observe the Sun's activities. There are 25 stations that are used for this purpose. Radio Frequency Interference (RFI) is a major obstacle when performing observation with CALLISTO. We have confirmed at least 2 stations out of 10 stations with a complete overview spectral (OVS) made available to us showed clear detection of these consistent types of RFI for each specific region. In Malaysia, these RFI are also clearly detected. The major RFI affecting CALLISTO within radio astronomical windows below $1 \mathrm{GHz}$ are local electronic system specifically radio navigation (at $73.1 \mathrm{MHz}$ and $75.2 \mathrm{MHz}$ ), broadcasting (at (i) $151 \mathrm{MHz}$, (ii) $151.8 \mathrm{MHz}$ and $152 \mathrm{MHz}$ ), aeronautical navigation (at (i) 245.5 $\mathrm{MHz}$, (ii) $248.7 \mathrm{MHz}$ and (iii) $249 \mathrm{MHz}$ and fixed mobile at (i) $605 \mathrm{MHz}$, (ii) $608.3 \mathrm{MHz}$, (iii) 612.2 $\mathrm{MHz}$, (iv) $613.3 \mathrm{MHz}$ ). It is obviously showed that all sites within this region are free from interference at $320-330 \mathrm{MHz}$ and is the best specific region to be considered for solar burst monitoring. We also investigate the effect of RFI on detection of solar burst. We have considered type III solar bursts on $9^{\text {th }}$ March 2012 in order to measure the percentage of RFI level during the solar burst. The RFI level is as low as $6.512 \%$ to $80.769 \%$ above solar burst detection.
\end{abstract}

Keywords: CALLISTO; low frequency; solar burst, type III; Radio Frequency Interference (RFI)

\section{INTRODUCTION}

The CALLISTO (Compound Astronomical Low cost Low frequency Instrument for Spectroscopy and Transportable Observatory) was considered as a global network of spectrometer system with the purpose to observe the Sun's activities located in different regions of the Earth [1]. The spectrograph is a new concept for solar radio spectrographs, designed by ETH Zurich [2]. ETH Zurich has developed a number of solar radio spectrometers since 1980s [3,4]. It is planned to carry out co-ordinated radio spectral 
observations of the solar corona from various locations around the world during the International Heliophysical Year 2007 [5]. Up to date, within five years there are 25 stations that are used for this purpose. The network consists of a number of spectrometers located around the globe, and designed to monitor solar radio emission in the meter and decametre bands [6]. This is important because radio waves at the meter and decimeter waves can be used as a diagnostics of the solar processes [7]. Meanwhile, solar space weather events like coronal mass ejections and solar flares are usually accompanied by solar radio bursts, which can be used for a low-cost real-time space weather monitoring [8]. Unfortunately, the flux density of low-frequency radio bursts has rarely been observed over a large range of frequency [9]. Therefore study in this region is important. It is believed that numerous bursts observed in space commence below $10 \mathrm{MHz}$, sometimes below $1 \mathrm{MHz}$ [10]. Ideally, radio signals are characterized by complex variability patterns including both non-stationarities and non-linearities [11]. The solar active region provides nonlinear electron acceleration or injection processes and the properties of the complex ambient coronal structures [12]. In Malaysia, the study of solar burst is just in beginning phase. Malaysia become as a $19^{\text {th }}$ host station for this project. We make an effort by joining e-CALLISTO research network. This data is retrieved automatically from currently 21 sites. Here, we possible to monitor Sun's activities within 12 hours throughout a year $[13,14]$. Daily observing period is starting from 11:30 to 23:30 UT. Generally, the main objective of this research is to observe the solar radio spectrum for 24 hours per day through all the year.

However, an increasing use of technologies, especially in wireless system as a mode of communication require us to monitor RFI that might affect solar signal detection in radio region. Man-made radio signals occur throughout the radio spectrum, are variable in time and frequency, and can be strong enough to be detected in the far-out side lobes of the antenna primary beam response [15]. RFI presents a significant limitation on the ability to detect and characterize pulsed emission [16]. This is an issue that needs to be concerned. To solve this problem, a wide range of methods for identification and mitigation of RFI has been considered [17] including post-correlation matrix projection methods [18,19], blanking [20]) and coherent subtraction [21]. Therefore, in this work, we presented the initial results of a spectral overview of selected sites and compare with our site [22]. We provide these details of the interference sources to as a plan to choose the best data at certain region [23-38].

\section{II. METHODOLOGY AND DATA ANALYSIS}

CALLISTO spectrometer is a programmable heterodyne receiver which has been setup at the National Space Centre; Selangor on 22nd February 2012 is a valuable new tool location for monitoring solar activity and ideal for space weather research. We used a log periodic dipole antenna (LPDA) directly connected via low loss coaxial cable to the CALLISTO spectrometer. By constructing and understanding the principle of the log dipole periodic antenna and then connect it to the CALLISTO spectrometer as receiver, some solar activities observations such as solar flares and Coronal Mass Ejections (CMEs) can be done [23-25]. The antenna has a boom length of 5.45 meter and covers the range from $45 \mathrm{MHz}$ till $870 \mathrm{MHz}$ with gain of about $7 \mathrm{dBi}$. A low noise preamplifier is connected to maximize the gain more than $10 \mathrm{~dB}$ compensate for cable loss and to compensate higher noise figure of the receiver of about $9 \mathrm{~dB}$. So far, wideband frequency techniques have focused on optimizing the accuracy and dynamic range achievable in the continuous burst by divided it into 15 minutes for each image to gain higher resolution of frequency. Each spectrum data file and the tracking log file 
stored by CALLISTO software and tracking software are transferred to the data acquisition server to process and display data and the current tracking status in the Space Weather Monitoring Laboratory (SWML) in real time.

Table 1. Geographical coordinates of the National Space Centre, Selangor, Malaysia.

\begin{tabular}{cc}
\hline Coordinate & Value \\
\hline Latitude & $3.0833^{\circ} \mathrm{N}$ \\
Longitude & $101.5333^{\circ} \mathrm{E}$ \\
Height & $150 \mathrm{~m}$ \\
Local Time & GMT $+08 \mathrm{~h}$ \\
\hline
\end{tabular}

A survey has been done in a day starting from 9.00 am till $17.00 \mathrm{pm}$ on $21^{\text {st }}$ of February 2012. We also compare the RFI signal from our site with Thailand observatory as a part of our research [26]. Measurement of the quiet sun is called ON-source while the measurement away from the sun is called the OFF - source. We then identified the continuous signal of frequency responses from the spectrum analyzer. Data will be transferred to a computer for further analysis. Objectively, this measurement involves technical basis to decide how to continue concerning spectroscopic measurements below $870 \mathrm{MHz}$. Detailed analyses also revealed the interference sources that caused an interruption of solar signal. In order to achieve a good signal of the Sun with high signal to noise ratio (SNR), it is necessary to recognize the range of minimum interference. We need to identify the signal sources, we then compared with the reference of frequency allocation of the International Telecommunication (ITU) and Malaysian Communication and Multimedia Commission (MCMC) as well.

\section{RESULTS AND ANALYSIS}

Radio Frequency Interference (RFI) is a major obstacle when performing observations with CALLISTO [27]. In order to study in detail the effect of RFI in solar monitoring, we divided the selected frequency into six subsections to investigate in details application of technologies at specific frequencies. At the same time, we can select the best site that possible gives a good solar burst data. This is very important because it is not easy to eliminate a dominant and strong signal if the burst is occurring in that particular region. Table 1 shows the RFI affects CALLISTO within radio astronomical windows. The entire set of observations is taken from different sites of CALLISTO network. Here, we focused on specific frequencies to observe any interference sources that possibly obstruct the Sun's signal. We are now in the process of analyzing an RFI in solar burst. The observations performed on 9th March 2012 at 4.24 UT. The solar burst type III is detected with a fine structure as illustrated in Figure 1. Some site show quite clearly a type III radio burst; however, there are also other signals in the spectra. 


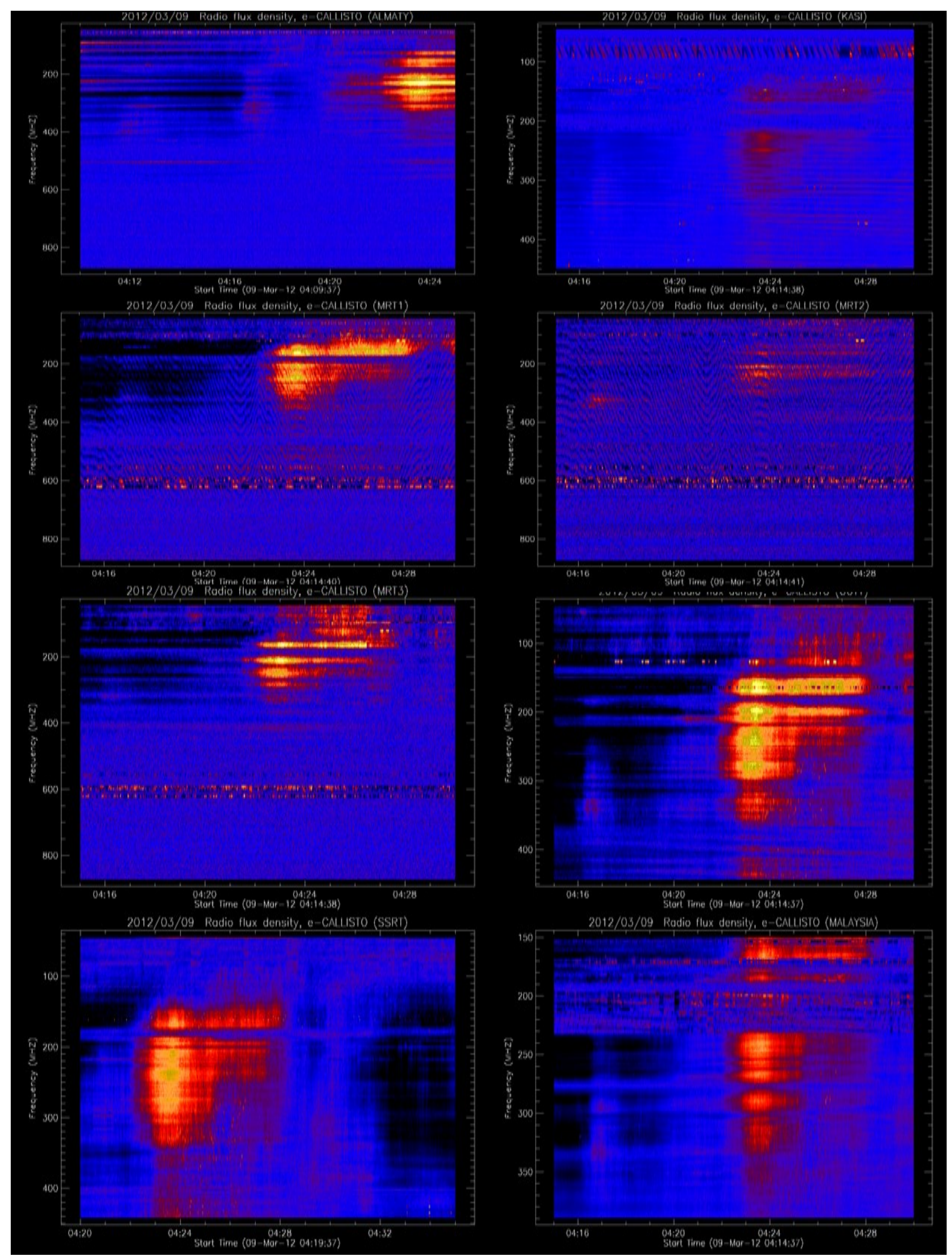

Figure 1. Solar burst type III detected at all sites from e-Callisto network on 9th March 2012 at 4.24 UT. Figure: Solar burst type IV detected at (i) Almaty, Kazasthan (ii) Kasi, Korea (iii) MRT1, Mauritius, (iv) MRT2, Mauritius (v) MRT3, Mauritius (vi) Ooty, India (vii) SSRT (viii) Angkasa, Malaysia. 
Not all sites can observe this burst due to the visibility of the sun above horizon period of observations factor. Therefore, different places are important to confirm and select a best data. Furthermore, we should accept that radio frequency measurements are almost always subject to some level of radio frequency interference (RFI) under background noise. At the first stage of calibration, we have subtracted the background signal from the CALLISTO data by using RAPP software. The entire CALLISTO system runs automatically, producing a daily report of the site events and coincidence detections. Data acquisition and all other activities at each site are controlled by a standard computer. Each 15 minutes, the previous data are uploaded to the workstation where they are processed and archived.

It must be emphasized that there are different types of interference unpredictable, wellknown interference, permanently and non-permanently RFI [28]. From the present data one may derive several predictions. Based on the spectral overview, a lot of military satellites were detected between $190 \mathrm{MHz}$ and $270 \mathrm{MHz}$. The signals are thought to be due to a local oscillator at $296 \mathrm{MHz}$. Several bands of spectral ranges are almost free from interferences. We can clearly observe that in the range of $300 \mathrm{MHz}$ till $400 \mathrm{MHz}$, this part of the spectrum is very clean and undisturbed. In addition, we can also use the range from 250 till $270 \mathrm{MHz}$, although there is a moderate interference. All reserved frequencies are still free from interference. Unfortunately, most of the strong and fluctuate interferences are home made by local electronic devices and local oscillators and clearly been seen from $85 \mathrm{MHz}$ till 150 MHz. However, it would be difficult or impossible to eliminate it completely. Due to the results, three frequency ranges can be identified for useful astronomical observations (i) between $45 \mathrm{MHz}$ and $80 \mathrm{MHz}$ (ii) between $240 \mathrm{MHz}$ and $380 \mathrm{MHz}$ and (iii) between 780 $\mathrm{MHz}$ and $850 \mathrm{MHz}$.

\section{CONCLUSIONS}

Investigation on the role of interference caused by human made technologies at low frequency region 45-870 MHz has been discussed. Radio Frequency Interference (RFI) gives a great impact in in solar radio astronomical field. The cross modulation of signal received by using a log periodic dipole antenna or a telescope with human-made technologies signal will influence the significant data. However, the radio frequency interference region could be identified by setting up a RF interference survey at the chosen location. We can conclude that Ooty, India is the best site to observe solar monitoring. Nevertheless, other sites also could be possible to detect the best time of solar events. The e-CALLISTO network is growing with potential solar burst candidate data. One should not neglect is that Malaysia can be possibly become a good site for solar monitoring due to 12 hours monitoring. This indicates a good percentage of continuums bursts that last for a few hours to days. However, the effort of minimizing the interference needs to be done, so that we would be able to obtain reliable and significant solar burst data.

It is hope that by studying the RFI in solar monitoring at low frequency from eCALLISTO network data, we could possibly choose the best solar burst data for analysis. This analysis would be possible to help others to choose a data that have minimum or without RFI at certain region of frequency from different sites. This is one of the initial results that need to be extended for future. Yet, it might possible that there will be an increasing of quality data by improving the CALLISTO configuration system itself (larger antenna with smaller main beam) or by solving the method to reduce the interference technically. 


\section{ACKNOWLEDGEMENT}

We are grateful to LASCO,SDO/AIA, NOAA and SWPC make their data available online. This work was partially supported by the FRGS (600 RMI/FRGS 5/3 2012) UiTM grants. Special thanks to the National Space Agency and the National Space Centre for giving us a site to set up this project and support this project. Solar burst monitoring is a project of cooperation between the Institute of Astronomy, ETH Zurich, and FHNW Windisch, Switzerland, MARA University of Technology and University of Malaya. This paper also used NOAA Space Weather Prediction Centre (SWPC) for the sunspot, radio flux and solar flare data for comparison purpose. The research has made use of the National Space Centre Facility and a part of an initiative of the International Space Weather Initiative (ISWI) program.

\section{BIOGRAPHY}

Dr. Zety Sharizat Hamidi is currently a lecturer and focused in Solar Astrophysics research specifically in radio astrophysics at the School of Physics and Material Sciences, Faculty of Sciences, MARA University of Technology, 40450, Shah Alam, Selangor, Malaysia. Involve a project under the International Space Weather Initiative (ISWI) and also a lecturer in School of Physics and Material Science, at MARA University of Technology, Shah Alam Selangor.

Dr. Nur Nafhatun Md Shariff is a senior lecturer at 2Academy of Contemporary Islamic Studies (ACIS), MARA University of Technology, 40450, Shah Alam, Selangor, MalaysiaHer current research is more on sustainability; environmental aspect. She is looking forward for cross-field research, i.e. solar astrophysics, light pollution measurement (mapping) and religious studies.

C. Monstein is a senior Engineer at Institute of Astronomy, Wolfgang-Pauli-Strasse 27, Building HIT, Floor J, CH-8093 Zurich, Switzerland and one of the researchers who initiated the CALLISTO system around the world.

\section{References}

[1] C. M. O. Benz Arnold, H. Meyer, Callisto, a New Concept for Solar Radio Spectrometers, Kluwer Academic Publishers, 2004.

[2] A. O. Benz, Monstein, C., Meyer, Solar Physics 226 (2005).

[3] A. O. Benz, Guedel, M., Isliker, H., Miszkowicz, S., Stehling, W., Solar Physics 133 (1991).

[4] P. Messmer, Benz A. O., Monstein C., Solar Physics 187 (1999) 335-345.

[5] R. R. C. Monstein, C. Kathiravan, Bull. Astr. Soc. India 35 (2007) 473-480.

[6] A. O. Benz, et al., Earth Moon and Planets 104 (2009) 8.

[7] N. R. L. D. J. McLean, Studies of Emission from the Sun at Metre Wavelengths, Cambridge University Press, Cambridge, 1985.

[8] V. V. Lobzin, Cairns I. H., Robinson P. A., Steward G., Patterson G., Automatic recognition of type III solar radio bursts: automated radio burst identification system method and first observations, Space Weather (2009).

[9] W. C. E. G. A. Dulk, R. Manning, J.-L. Bougeret, Astronomy \& Astrophysics 365 (2001) 6.

[10] G. A. Dulk, Type III solar radio bursts at long wavelengths, in: E.W.M.G. R. Stone (Ed.), Proc. Chapman Conference on Space-Based Radio Observations at Long Wavelengths, Geophys. Monogr., 2000. 
[11] R. R. R. T. B. Veronese, M. J. A. Bolzan, F. C. Rocha Fernandes, H.S. Sawant, M. Karlicky, Journal of Atmospheric and Solar-Terrestrial Physics 73 (2011) 4.

[12] A. Nindos, Aurass, H., Klein, K.-L., Trotter, G., Solar Physics 253 (2008) 41.

[13] Z. S. Hamidi, N. Shariff, Z. Abidin, Z. Ibrahim, C. Monstein, Middle-East Journal of Scientific Research 12 (2012) 6.

[14] Z. S. Hamidi, S. Chumiran, A. Mohamad, N. Shariff, Z. Ibrahim, N. Radzin, N. Hamzan, N. Anim, A. Alias, American Journal of Modern Physics 2 (2013) 4.

[15] S. W. Ellingson, Radio Science 40 (2005).

[16] S. V. W. John Hogden, Georey C. Bower, Sarah Michalak, Andrew Siemion, Daniel Werthimer, Comparison of RFI Mitigation Strategies for Dispersed Pulse Detection, ApJ (2012).

[17] W. Baan, RFI Mitigation Workshop, 2010.

[18] A. Leshem, van der Veen A., Boonstra A., ApJS 131 (2000).

[19] J. Kocz, Briggs F., Reynolds J. , AJ 140 (2010).

[20] J. S. Deneva, Cordes J. M., McLaughlin M. A., et al. , ApJ 703 (2009).

[21] S. W. Ellingson, Hampson, G. A., ApJS 147 (2003).

[22] Z. S.Hamidi, Z. Abidin, Z. Ibrahim, C. Monstein, N. Shariff, International Journal of Fundamental Physical Sciences 2 (2012) 32-34.

[23] Z. S.Hamidi, et al., International Journal of Fundamental Physical Sciences 2 (2012) 4.

[24] Z. S. Hamidi, Z. Abidin, Z. Ibrahim, N. Shariff, C. Monstein, Observations of coronal mass ejections (CMEs) at low frequency radio region on 15th April 2012, in: R. Shukor (Ed.), PERFIK 2012, American Institute of Physics, Malaysia, 2013, pp. 5.

[25] Z. S. Hamidi, N. Anim, N. N. M. Shariff, Z. Z. Abidin, Z. A. Ibrahim, C. Monstein, Dynamical structure of solar radio burst type III as evidence of energy of solar flares, in: R.Shukor (Ed.), PERFIK 2012, American Institute of Physics, Malaysia, 2013, pp. 11-15.

[26] R. Umar, et al., Middle East Journal of Scientific Research 14 (2013) 861-866.

[27] Z. S. Hamidi, Z. Z. Abidin, Z. A. Ibrahim, N. N. M. Shariff, U. F. S. U. Ibrahim, R. Umar, Preliminary analysis of investigation Radio Frequency Interference (RFI) profile analysis at Universiti Teknologi MARA, IEEE, 2011, pp. 311-313.

[28] Z. S. Hamidi, Z. Z. Abidin, Z. A. Ibrahim, N. N. M. Shariff, AIP Conference Proceedings 1454 (2012) 43.

[29] Z. S. Hamidi, N. N. M. Shariff, C. Monstein, W. N. A. Wan Zulkifli, M. B. Ibrahim, N. S. Arifin, N. A. Amran, International Letters of Natural Sciences 5 (2014) 10-17.

[30] Z. S. Hamidi, N. N. M. Shariff, International Letters of Chemistry, Physics and Astronomy 4 (2014) 29-36.

[31] Z. S. Hamidi, N. N. M. Shariff, International Letters of Chemistry, Physics and Astronomy 5 (2014) 32-42. 
[32] Z. S. Hamidi, N. N. M. Shariff, International Letters of Chemistry, Physics and Astronomy 5 (2014) 43-49.

[33] Z. S. Hamidi, N. N. M. Shariff, International Letters of Chemistry, Physics and Astronomy 7 (2014) 21-29.

[34] Z. S. Hamidi, N. N. M. Shariff, International Letters of Chemistry, Physics and Astronomy 7 (2014) 30-36.

[35] Z. S. Hamidi, N. N. M. Shariff, C. Monstein, Z. A. Ibrahim, International Letters of Chemistry, Physics and Astronomy 7 (2014) 37-44.

[36] Z. S. Hamidi, N. N. M. Shariff, C. Monstein, W. N. A. Wan Zulkifli, M. B. Ibrahim, N. S. Arifin, N. A. Amran, International Letters of Chemistry, Physics and Astronomy 8 (2014) 13-19.

[37] Z. S. Hamidi, N. N. M. Shariff, C. Monstein, W. N. A. Wan Zulkifli, M. B. Ibrahim, N. S. Arifin, N. A. Amran, International Letters of Chemistry, Physics and Astronomy 9 (2014) 8-15.

[38] Z. S. Hamidi, N. N. M. Shariff, M. F. Ali, C. Monstein, W. N. A. Wan Zulkifli, M. B. Ibrahim, N. S. Arifin, N. A. Amran, International Letters of Chemistry, Physics and Astronomy 9 (2014) 84-92. 